\title{
Applying joint theoretical experimental research to aptamer modeling
}

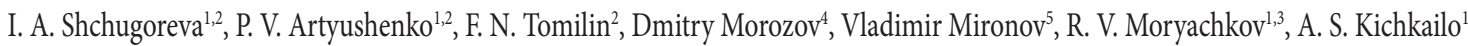

${ }^{1}$ Laboratory for Digital Controlled Drugs and Theranostics, Federal Research Center "Krasnoyarsk Science Center SB RAS", Krasnoyarsk 660036, Russian

Federation

${ }^{2}$ Department of Chemistry, Siberian Federal University, Krasnoyarsk 660041, Russian Federation

${ }^{3}$ Laboratory of Physics of Magnetic Phenomena, Kirensky Institute of Physics, Krasnoyarsk 660012, Russian Federation

${ }^{4}$ Nanoscience Center and Department of Chemistry, University of Jyväskylä, Jyväskylä 40014, Republic of Finland

${ }^{5}$ Department of Chemistry, Lomonosov Moscow State University, Moscow 119234, Russian Federation

The aim of the research. In this work we studied the structure of LC-18 DNA aptamer, which exhibits specific binding to lung adenocarcinoma cells. Obtaining the 3D structure of the aptamer is necessary for understanding the mechanism of binding of the aptamer to the target. Therefore, the aim of the research was modeling of the LC-18 aptamer spatial structure using combination of theoretical methods: DNA folding tools, quantum-chemical calculations and molecular dynamic simulations.

Material and methods. The secondary structure of the LC-18 aptamer was predicted by using OligoAnalyzer and MFold online software under the conditions typical small-angle X-ray scattering (SAXS) experiment. The molecular modeling of the aptamer was carried out using the Avogadro program. For prediction of the structure two computational methods were used: quantum-mechanical method with third-order density-functional tight-binding (DFTB3) and molecular dynamics (MD) with force fields.

Results. In this paper it was shown that molecular simulations can predict structures from the SAXS experiments. OligoAnalyzer and MFold web servers have been used to generate a set of several likely models. However, more accurate calculations have showed that these models do not predict the relative importance of isomers. Meanwhile, application of quantum-chemical and molecular dynamics calculations have showed reliable molecular structures which have a small deviations from the experimental SAXS curves.

Conclusion. This study demonstrates the approach for modeling 3D structures of DNA-aptamers in solution using both experimental and theoretical methods. It could be very helpful in designing more efficient aptamers based on results obtained from molecular simulations.

Key words: LC-18, DNA aptamer, lung adenocarcinoma, SAXS, DFTB3.

Conflict of interest. The authors declare the absence of obvious and potential conflicts of interest associated with the publication of this article.

Citation: Shchugoreva IA, Artyushenko PV, Tomilin FN, Morozov D, Mironov V, Moryachkov RV, Kichkailo AS. Applying joint theoretical experimental research to aptamer modeling. Siberian Medical Review. 2021; (2):105-106. DOI: 10.20333/2500136-2021-2-105-106

Aptamers are synthetic RNA or DNA oligonucleotide ligands capable of recognizing and binding with other molecules. Aptamer-target interactions depend on the aptamer's spatial structure, distribution of its charges, and the nature of its binding molecules. However, obtaining the $3 \mathrm{D}$ structure is a difficult task. The approach suggested in this work is to apply joint theoretical-experimental research to aptamer modeling.

Using the small-angle X-ray scattering (SAXS), the aptamer was investigated in solution at temperatures of $5^{\circ} \mathrm{C}$ and $20^{\circ} \mathrm{C}$. As a result of the experiment, the shape of the molecule in solution was obtained. The LC-18 aptamer consists of 35 nucleotides. To predict its secondary structures, the online servers for folding DNA molecules OligoAnalyzer [1] and Mfold [2] were used. The simulation was carried out under conditions of the SAXS experiment: in the presence of ions $\mathrm{Na}^{+} 146 \mathrm{mM}$ and $\mathrm{Mg}^{2+} 0.5 \mathrm{mM}$ at $5{ }^{\circ} \mathrm{C}$ and $20^{\circ} \mathrm{C}$. Four models were chosen for further investigation as the most probable secondary structures with the lowest energy, moreover Model- 1 according to both programs is the most likely secondary structure of LC-18 (fig.).

The corresponding tertiary structures of Model-1, Model-2, Model-3, and Model-4 were prepared using the Avogadro and Facio [3] programs and optimized with the FMODFTB3/PCM method. The distribution of LC-18 structures in the order of increasing their total energy after optimization: Model-4 $\rightarrow$ Model-1 $\rightarrow$ Model-3 $\rightarrow$ Model-2, which differs from the distribution in MFold and OligoAnalyzer. Comparison of the molecules geometry with the shape obtained by SAXS showed the greatest agreement for the Model- 4 model.

For the optimized structures, molecular dynamics (MD) calculations were performed using the Gromacs 2019.8 [4] software package with the Amber14sb force field and the TIP3P model for water. As a result of simulations (200 ns at $310 \mathrm{~K}$ ), the initial geometry of the molecules changed, most significantly for the Model-1, Model-2, Model-4 models. A comparison of the structures obtained after MD with the SAXS shape showed a high convergence of the geometry with the Model-3 model, better than for the Model-4 model before molecular dynamics. The calculations have shown that geometry optimizations using both the quantum-chemical and molecular dynamics methods produce structures that are very similar to the experimental SAXS curves with small deviations.

Thus, the combination of computer simulations and SAXS methods allows to select the secondary structure of the aptamer from a variety of options offered by the servers for folding. It also allows to identify the conformation of the molecule that is closest to the conformation of the aptamer in solution. 


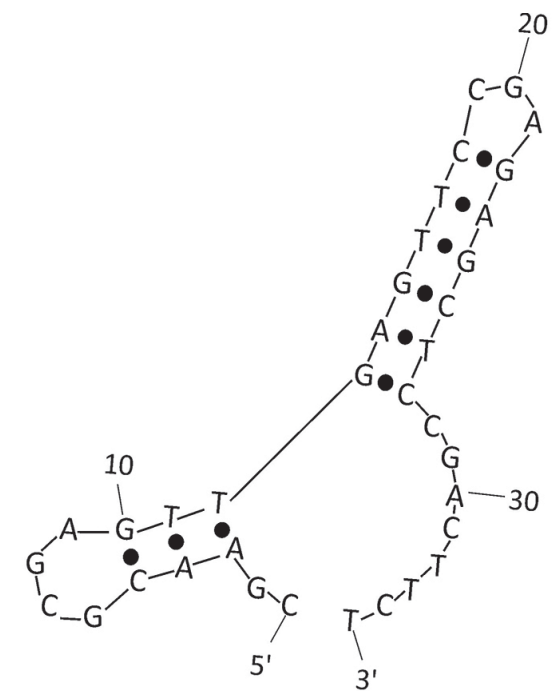

Model-1

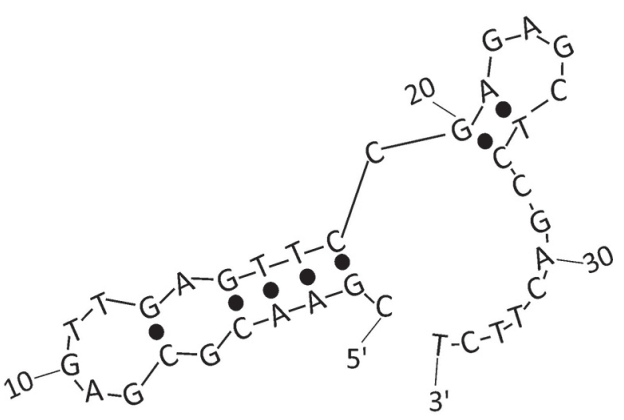

Model-2

20

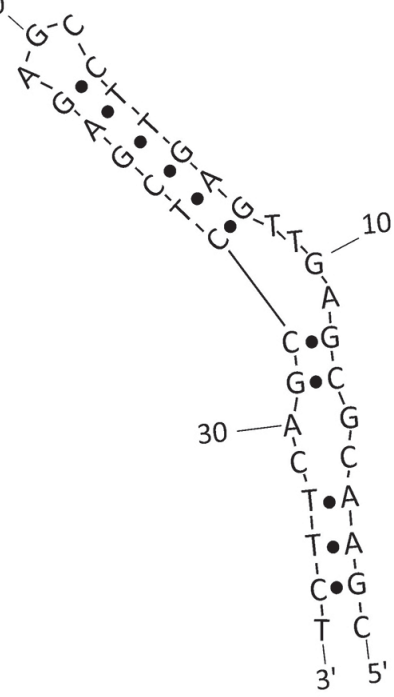

Model-4

30

Model-3

Figure. Secondary structures of LC-18 aptamer models.

\section{References}

1. Owczarzy R, Tataurov AV, Wu Y, Manthey JA, McQuisten KA, Almabrazi HG, Pedersen,KF, Lin Y, Garretson J, McEntaggart NO. IDT SciTools: a suite for analysis and design of nucleic acid oligomers. Nucleic Acids Research. 2008; (36):163-169.

2. Zuker M. Mfold web server for nucleic acid folding and hybridization prediction. Nucleic Acids Research. 2003; (31):3406-3415.

3. Suenaga M. Facio: New Computational Chemistry Environment for PC GAMESS. Journal of Computer Chemistry. 2005; (4):25-32.

4. Abraham MJ, Murtola T, Schulz R, Páll S, Smith JC, Hess B. and Lindah E. Gromacs: High performance molecular simulations through multi-level parallelism from laptops to supercomputers. SoftwareX, 2015; 1-2:19-25.

\section{Author information}

Irina A. Shchugoreva, Junior researcher, Laboratory for Digital Controlled Drugs and Theranostics, Federal Research Center "Krasnoyarsk Science Center SB RAS"; Address: 50, Akademgorodok, Krasnoyarsk, Russian Federation 660036; Department of Chemistry, Siberian Federal University; Address: 79, Svobodny pr, Krasnoyarsk, Russian Federation 660041; Phone: +79029691154; e-mail: shchugorevai@mail.ru, https://orcid.org/0000-0003-4207-1627

Polina V. Artyushenko, Candidate of sciences in physics and mathematics, senior researcher, Laboratory for Digital Controlled Drugs and Theranostics, Federal Research Center
"Krasnoyarsk Science Center SB RAS"; Address: 50, Akademgorodok, Krasnoyarsk, Russian Federation 660036; Department of Chemistry, Siberian Federal University; Address: 79, Svobodny pr, Krasnoyarsk, Russian Federation 660041; Phone: +79082005639; e-mail: art_polly@mail.ru, https://orcid.org/0000-0002-3176-8202

Felix N. Tomilin, Candidate of Sciences in Chemistry, associate professor, Department of Chemistry, Siberian Federal University; Address: 79, Svobodny pr, Krasnoyarsk, Russian Federation 660041; Phone: +7-950-978-88-90; e-mail: felixnt@gmail.com, https://orcid. org/0000-0002-3578-6141

Dmitry I. Morozov, Candidate of Sciences in Chemistry, Postdoctoral researcher, Nanoscience Center and Department of Chemistry, University of Jyväskylä; Address: PO Box 35, Jyväskylä, Republic of Finland 40014; Phone: +358-14-260-12-11 e-mail: dmitry.morozov@ jyu.fi, https://orcid.org/0000-0001-9524-948X

Vladimir A. Mironov, Candidate of sciences in physics and mathematics, senior research er, Department of Chemistry, Lomonosov Moscow State University; Address: Leninskie Gory, Moscow, Russian Federation 119234; Phone: +7 (495) 939-21-75; e-mail: vladimir.a.mironov@ gmail.com, https://orcid.org/0000-0002-9454-5823

Roman V. Moryachkov, researcher, Laboratory for Digital Controlled Drugs and Theranostics, Federal Research Center "Krasnoyarsk Science Center SB RAS"; Address: 50, Akademgorodok, Krasnoyarsk, Russian Federation 660036; Laboratory of Physics of Magnetic Phenomena, Kirensky Institute of Physics; Address: 50/38, Akademorodok, Krasnoyarsk, Russian Federation 660012; Phone: +7-913-511-38-16; e-mail: romanautilus@gmail.com, https://orcid.org/00000002-0409-779X

Anna. S. Kichkailo, Doctor of Sciences in Biological, leading researcher, Laboratory for Digital Controlled Drugs and Theranostics, Federal Research Center "Krasnoyarsk Science Center SB RAS"; Address: 50, Akademgorodok, Krasnoyarsk, Russian Federation 660036; Phone: +7903923-8402; e-mail: annazamay@yandex.ru, https://orcid.org/0000-0003-10544629

Дата поступления: 16.02.2021 Дата речензирования: 18.03 .2021 Принята к печати: 31.03 .2021 Received 16 February 2021 Revision Received 18 March 2021 Accepted 31 March 2021 